\title{
RETOS Y PERSPECTIVAS PARA UNA FORMACIÓN BIOÉTICA EN LOS ESTUDIANTES DEL ÁREA EMPRESARIAL
}

\author{
CHALLENGES AND PROSPECTS FOR BIOETHICS FORMATION FOR \\ STUDENTS IN THE BUSINESS AREA \\ ANA María Gutiérrez HubY* \\ Docente Principal de la Facultad de Ciencias Contables
}

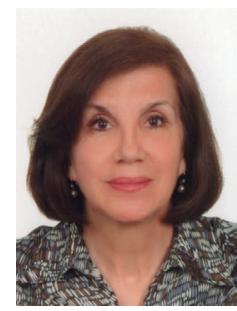

Universidad Nacional Mayor de San Marcos-UNMSM / Lima-Perú

[Recepción: Setiembre de 2014/ Conformidad: Octubre 2014]

\section{RESUMEN}

Este artículo aborda la bioética desde un contexto educativo. Se plantea la necesidad de incorporarla como una disciplina aplicada dentro de la educación empresarial, para que oriente el establecimiento de principios y que la acerquen a situaciones relacionadas con la vida humana. Entre los temas abordados encontramos conceptos y fundamentos de la bioética y su relación con las funciones de la empresa, el rol del Estado como garante del derecho ciudadano a la educación y a la construcción de una sociedad justa y humanizada, razones del por qué incluirla como disciplina en la currícula universitaria, competencias que se desarrollarán en los estudiantes, los aspectos que comprende la formación de formadores, metodologías y estrategias para el diseño curricular, retos y perspectivas que se presentan para la formación en bioética. Se arribó a las siguientes conclusiones: A nivel institucional no existe coherencia en la misión con el desarrollo de un perfil bioético en los estudiantes. Los planes de estudio del área empresarial no contemplan la bioética en la malla curricular, como consecuencia, se refleja un bajo nivel de conocimiento de este concepto en los estudiantes.

Palabras clave:

Educación; empresa; malla curricular; estrategias.

\begin{abstract}
This article addresses in bioethics from an educational context. The need to incorporate it as an applied discipline within enterprise education, to guide the establishment of principles and the approach to situations involving human life is posed. Among the topics covered are concepts and principles of bioethics and its relationship with business functions, the state's role as guarantor of citizen education law and building a just and humane society, reasons why include it as a discipline in university curriculum, develop competencies in students aspects comprising the training of trainers, methods and strategies for curriculum design, challenges and perspectives presented for training in bioethics. He reached the following conclusions: At the institutional level there is no consistency in the mission to develop bioethical profile students. The curricula of business area do not include bioethics in the curriculum; therefore, a low level of understanding of this concept is reflected in students.
\end{abstract}

Keywords:

Education; enterprise; curricular net; strategies

\footnotetext{
* Doctora en Administración. Magíster en Gestión Empresarial - UNMSM. Email: anamaria_01053@yahoo.es.

** Colaboradora: Mg Eugenia Amador Murguía. Docente de la Universidad de Guadalajara - Centro Universitario de Los Lagos.

*** Colaboradora: Elsa Violeta Rafael Díaz.
} 


\section{INTRODUCCIÓN}

Las características que definen a los modelos de convivencia actual en el orden político, económico y social, han dado lugar como producto de su expansión, impacto y aceptación en el medio, a una sociedad más tecnificada, materializada e individualista, que viene perdiendo el rumbo de su condición vital y su sentido como humanidad, en donde ha irrumpido culturalmente una condición transgresora por parte del ser humano, vislumbrándose entonces un horizonte poco claro en el que se destaca una preocupante crisis de valores que va cada vez en aumento, cambiando y afectando los roles de las familias y la de sus integrantes; en especial, de los más jóvenes.

Es este contexto, en el que se viene desenvolviendo la sociedad actual, experimentando un proceso de transformación, siendo tiempos donde predomina el saber hacer y las habilidades técnicas por sobre los saberes teóricos, en donde el pragmatismo privilegia las habilidades procedimentales sobre las actitudinales, en el que el valor del saber productivo prima sobre otros saberes, en donde las medidas se orientan por estándares basados en la eficiencia y en los resultados más que en los procesos y en donde la búsqueda del éxito y el bienestar, se basan en modelos de alta competitividad.

Es en este marco donde tiene cabida la bioética, disciplina que ha asumido el estudio sistemático de la conducta humana en el ámbito de las ciencias de la vida y la salud a la luz de principios que la fundamentan, la cual encuentra en la educación un extraordinario medio para la formación y transmisión de su propósito, el cual es de interrogar y dilucidar temas esenciales de la vida humana como son: su dignidad, el sufrimiento y enfermedad, las relaciones del hombre con los otros seres vivos y con el ambiente, buscando transformar el sentido de la proyección tecnocientífica que viene generando un nuevo contexto de emociones y formas de vida novedosas, que aunque algunas de ellas puedan aportar a la calidad de vida, también pueden fomentar el dolor, el sufrimiento, el sinsentido y la exclusión.

Dado que la educación es considerada por la bioética un vehículo para sus fines de adoctrinamien- to que se menciona, es preciso reflexionar sobre el papel que ha venido desempeñando la educación y en particular, la educación universitaria, en este proceso de transformación, considerando que ella también se ha visto afectada por el impacto de los cambios producidos; prueba de ello, es la reducción de materia en los currículos de los programas académicos, que contribuyen a la formación del hombre como el civismo, historia, ética, sociología y materias afines, sustituyéndolas por las ciencias, las tecnologías, las matemáticas, los idiomas extranjeros. Esta reflexión debe partir del sentido amplio que encierra el término educar, que es buscar la elevación del hombre a su máxima expresión, poniendo a su alcance los medios más adecuados para lograr su desarrollo, definición genérica de la naturaleza de la educación que tiene la misma vigencia cuando nos referimos a la educación universitaria con el agregado de que esta asume un carácter especial esencial al situarse en el seno de la racionalidad científica, donde se cultiva la ciencia a través de la aplicación científica y la teoría pura, con una apertura y comunicación con todas las vertientes del pensamiento.

A este respecto, es importante referir que importantes organizaciones han alzado sus voces convocando a la profunda reflexión sobre la situación coyuntural a nivel mundial y a una participación activa, en su transformación hacia una forma más justa o al menos más adecuada a las necesidades humanas.

UNESCO es ejemplo de ello, habiendo evidenciado su preocupación por la problemática referida, emitiendo a la actualidad tres Declaraciones Universales referidas a la Bioética en los últimos diez años, recomendando explícitamente la enseñanza de esta disciplina en todos los niveles educativos.

Sin embargo, esta recomendación según refiere Vidal, Susana (2012), a nivel latinoamericano no ha sido tan fácil de concretar en términos de una falta de establecimiento de consensos en torno a los contenidos y metodologías para la enseñanza de esta disciplina, por la complejidad de los cambios y las problemáticas antes referidas que han venido dificultando la tarea de desarrollar lineamientos pedagógicos y didácticos que posibiliten a la bioética ser materia de un consciente y crítico dominio social. 
En general, en el caso de la formación universitaria y con una connotación especial en las carreras de las ciencias empresariales, que es el área materia de interés para el presente artículo, se requiere ir más allá del desarrollo de competencias laborales y académicas, preocuparse por la preparación de profesionales que hagan de la bioética un elemento de su capacidad de gestión, que se traduzca en la construcción de espacios éticos en los ambientes donde estos se desempeñen, entendiendo que nuestra sociedad requiere de profesionales formados con rigor y disciplina académica, pero sobre todo con cualidades humanas y con el sentido ético que requiere su profesión, haciéndolo coherente con su práctica diaria.

Por lo expresado, el presente artículo pretende contribuir con la difusión de esta disciplina, brindando alcances para su aplicabilidad en la formación universitaria del área empresarial y para darle el tratamiento que le corresponde, partiendo de considerarla un elemento básico y fundamental en la formación integral de los educandos, planteándola como una necesidad en el desarrollo profesional y por lo tanto, en su formación.

\section{FUNDAMENTACIÓN TEÓRICA DE LA BIOÉTICA Y SU RELACIÓN CON LAS FUNCIONES DE LA EMPRESA}

Producto de su adaptación a los cambios sociales la empresa ha acogido como modelo de gestión para sus operaciones a la Responsabilidad Social Empresarial (RSE) con lo cual amplió su actuación, la que tradicionalmente estuvo en el plano económico, incorporando ahora dos dimensiones: social y ambiental, las cuales pasaron a ser atendidas a través de sus obligaciones legales y éticas.

Con la incorporación de estas dimensiones se empezó a advertir la presencia de un modelo que tendría en cuenta a las otras partes de la sociedad con intereses legítimamente válidos; las empresas comienzan a concientizarse del impacto de su accionar, decidiendo voluntariamente aportar al desarrollo económico, a la mejora de la calidad de vida de la comunidad donde se ubican y de la sociedad en su conjunto; es decir, una toma de conciencia de sí misma, de su ambiente y de su papel en él.
Bajo este enfoque de la RSE las operaciones empresariales adquieren aplicabilidad en las dimensiones teóricas de la empresa, lo cual implica agregar valor a sus actividades frente a la comunidad, cumplir con la legislación vigente, siendo responsable por los impactos de sus decisiones sobre la sociedad y el medio ambiente.

Dentro de este contexto empresarial, si aplicamos la bioética vemos que esta adquiere particular relevancia en la reflexión sobre la manera de cómo gestionar los negocios a través de sus principios de autonomía, no maleficencia, justicia, beneficencia.

Si bien, los principios bioéticos son aplicados a las personas, por analogía las empresas adquieren las mismas características, siendo responsables de sus propias acciones. El reto que se presenta es lograr que las relaciones entre ambos sean éticas.

El principio de autonomía supone una relación entre entes autónomos en los que las partes persiguen intereses propios respetando los ajenos, como ejemplo de la aplicabilidad de este principio bioético es la relación vendedor-comprador, donde el primero tiene la obligación de brindar toda la información relevante para que el segundo pueda tomar una decisión informada respecto a adquirir o no, el bien o servicio.

El principio bioético de no maleficencia alude a abstención de realizar acciones que puedan causar daño a otros. La aplicabilidad en el ámbito empresarial podría darse en las actividades comerciales donde una determinada empresa tiene la capacidad potencial de causarle daño a muchos individuos simultáneamente, los daños pueden ir desde económicos hasta implicar la pérdida de vidas por diversas razones como: productos defectuosos, condiciones laborales inadecuadas, contaminación ambiental.

El principio de beneficencia, dado que las empresas son partes integrales de la sociedad, tienen la obligación de hacer el bien a otros. Implica, que tienen la obligación de producir productos y servicios seguros e involucrarse en actividades que promuevan una mejor calidad de vida de la comunidad y su entorno circundante.

En el principio de justicia se destaca el hecho de dar a cada quien lo que le corresponde, disminuyendo las situaciones de desigualdad (ideológica, social, 
cultural, económica). Encuentra su aplicabilidad en las empresas que poseen y manejan recursos diversos por lo que su distribución debe ser justa. Por ejemplo, en las relaciones laborales no debería haber discriminación en el reclutamiento o promoción del personal. Entre los conflictos éticos que tienen lugar dentro de este ámbito se pueden encontrar, el lucro desmedido, contaminación ambiental, competencia desleal.

\section{EL ESTADO: GARANTE DEL DERECHO CIUDADANO A LA EDUCACIÓN Y A LA CONSTRUCCIÓN DE UNA SOCIEDAD MÁS JUSTA Y HUMANIZADA}

El Estado como unidad política organizada tiene la función de garantizar los grandes intereses sociales: el bienestar, la salud general y la protección de los derechos de los ciudadanos, constituyéndose estos en su responsabilidad social como Estado. Dentro de la protección de los derechos ciudadanos está principalmente, la educación y el de proveer los medios y recursos necesarios que posibiliten su acceso equitativo.

El contenido de la constitución del Estado hace precisiones en torno a los derechos ciudadanos y sus deberes con los que se persigue una sana convivencia.

Dicha responsabilidad pasa por determinar el contexto educativo y las instituciones que canalizan este derecho de los ciudadanos. La concreción de esta función se da a través del establecimiento de normas que regulan su creación y funcionamiento ya que se dedican a resolver necesidades sociales en todos los ámbitos y estratos socioeconómicos.

En suma, la constitución consolida la educación como otro derecho fundamental cuya responsabilidad recae de forma directa en las diferentes instituciones de educación, incluidas las universidades. Es decir, en esas condiciones el Estado se constituye en el protector de la libertad de direccionamiento filosófico de las universidades, de forma que se garantice el cumplimiento de su papel institucional frente a la sociedad. Uno de los parámetros de dicho direccionamiento es la Responsabilidad Social Universitaria, la cual debe constituirse en toda una estrategia a seguir por parte de ellas y no mantenerse simplemente como una declaración de voluntad de apoyo a acciones concretas.

48/ QUIPURAMAVOC | Vol. 22(42) 2014
En consecuencia, la responsabilidad social del Estado tiene una connotación bioética al garantizar la protección de la vida como derecho fundamental, esta concepción que se materializa como resultado de la democracia, como expresión de equidad y participación solidaria de personas, libertad individual sin discriminación de sexo, raza, religión, idioma, etc. y respeto por la libertad de pensamiento. En suma, el Estado a través de las normas hace precisiones en torno a los derechos y deberes de los ciudadanos frente a la sociedad. Esta perspectiva bioética del estado se concreta en sus principios, estando implícita su condición de garante de ellos, los cuales son: autonomía, beneficencia, no maleficencia y justicia.

Con respecto a la autonomía, le corresponde aprobar legislaciones sobre el consentimiento informado yla educación, pues solamente con educación, conocimiento y reflexión se podrá ejercer libremente la autonomía, siendo un elemento indispensable para mejorar la calidad de vida de los ciudadanos; por otra parte, los principios de no maleficencia y justicia demandan un mayor rol de protección hacia la persona mediante la legislación o políticas públicas que garanticen al individuo el real ejercicio de sus derechos y en definitiva, el respeto hacia la dignidad. Finalmente, como garante del principio de justicia debe administrarlo en beneficio de toda la población, mediante asignación equitativa de prioridades desde una realidad de escasez.

\section{CONSIDERACIONES QUE LEGITIMAN LA INCORPORACIÓN DE LA BIOÉTICA AL CURRÍCULO UNIVERSITARIO}

La incorporación de la bioética al currículo universitario encuentra su legitimización a través de uno de los aspectos misionales de la educación: La formación. Mediante ella, se ayudará al estudiante a situarse con madurez frente a los sistemas de valores presentes en el ámbito cultural.

Desde esta perspectiva institucional, la legitimación, demanda una justificación de corte pedagógico que implica brindar una enseñanza en bioética coherente con los propósitos educativos institucionales. En este sentido, se presenta a la bioética como portadora de una legítima contribución para el cumpli- 
miento de los fines educativos con una aportación y una metodología a aplicar, que resalta su cualidad formativa y humanizante.

\section{COMPETENCIAS QUE SE DESARROLLAN EN LOS ESTUDIANTES}

Esta situación plantea varios desafíos para los educadores en Bioética ligados a los valores que se promueven en estos cambios, pues todos conducen a una determinada visión del mundo y de la sociedad; a la vez, que cristalizan ciertas ideas en torno a lo que es correcto e incorrecto, promoviéndose así ciertos valores, aunque también, contravalores.

Hoy como nunca se actualiza la necesidad de participar en procesos de toma de decisiones complejas que son determinantes para la vida individual y social y que, sin lugar a dudas, están atravesadas por situaciones éticas poniéndose en juego numerosos valores.

Por esta razón, se requiere de competencias y saberes para participar y aportar a la resolución de conflictos de manera pacífica, tolerante y prudente, a través de una educación bioética, teniendo en cuenta los desafíos planteados por los cambios.

La tarea de educar en bioética tiene que ver con el desarrollo de competencias y para lograrlo se requiere de una metodología educativa especializada que incluye conocimientos, habilidades, actitudes, comportamiento y comunicación.

Entre las competencias que permite desarrollar la bioética, cabe mencionar:

- Competencias cognitivas y procedimentales: Permiten al educando encarar y elucidar los dilemas morales implícitos en el quehacer cotidiano del ejercicio de su profesión.

- Competencias actitudinales: Desarrolla actitudes de tolerancia, respeto y discusión racional con un perfil solidario, respetuoso de las diferencias, abierto a distintos puntos de vista y hábitos que privilegian el intercambio de ideas.

\section{ASPECTOS QUE COMPRENDE LA CAPACITACIÓN DE FORMADORES EN BIOÉTICA}

Entre los aspectos que comprende la capacitación de los docentes en bioética, cabe referirse a los siguientes:

- Contenidos: Concientización del docente en la importancia de los contenidos y objetivos (procedimentales y actitudinales). Es imprescindible para generar un espíritu reflexivo, crítico, cuestionador y respetuoso en el alumno.

- Diálogo: Estimula en los alumnos el respeto por las opiniones ajenas durante el abordaje de temáticas controversiales y dilemáticas. Ello, conlleva a resaltar y potenciar el carácter solidario de la plana docente, partiendo de ellos esta conducta para impartirla.

- Homogeneidad: Homogenización de conocimientos y actitudes docentes para así obtener mejores resultados en el alumnado los que, por primera vez, transitan en el campo bioético.

- Discursividad: Capacidad del docente para expresar lo conocido. Se considera también la adecuada dicción, buen manejo del auditorio, correcta utilización de materiales didáctico-pedagógicos y su desenvolvimiento en clase.

- Entrenamiento: La práctica previa en las aulas por ayudantes, garantiza solvencia y seguridad y prepara al educador frente a posibles situaciones conflictivas que pueden presentarse en clase.

- Trabajo de campo: Es promover en el futuro docente, el desarrollo de una práctica pedagógica de manera más acabada y concreta que le permitirá experimentar como observador la situación real del trabajo a futuro.

\section{METODOLOGÍAS Y ESTRATEGIAS PARA EL DISEÑO CURRICULAR}

Para la inserción de la bioética en la educación se han postulado varias propuestas, unas que proponen que sea impartida de manera permanente abarcando todos los niveles de la educación, empezando desde la educación inicial; otras, que sea transversal y, otras 
por área contemplándola como una asignatura adicional; ello, finalmente, es una decisión que asumirán quienes dirigen la institución educativa.

Habiendo la necesidad de asegurar un desarrollo integral en los alumnos, además de las propias expectativas de la sociedad, la gran mayoría coincide en referir que hay la exigencia de abordar un currículum que no se limite sólo a una adquisición de conceptos y conocimientos académicos vinculados con la enseñanza tradicional sino, que, este incluya otros aspectos que contribuyan al desarrollo de las personas como son las habilidades prácticas, las actitudes y los valores.

El desarrollo de los contenidos bioéticos en el aula, exigen conocimientos específicos. En caso la inserción de la bioética fuera contemplada como un área educativa, se requeriría de docentes especializados en la materia; si fuera transversal exigiría un reciclaje en la mayoría de los docentes, los que tratarían dichos contenidos en sus respectivas asignaturas; es así que, en general se requerirían docentes con algunos requisitos mínimos como:

Mentes abiertas a la opinión de otros, disposición activa para el diálogo, respeto por las ideas de los demás, tolerancia hacia las elecciones, valores y principios que rigen los objetivos de vida buena de otros individuos y comunidades, prudencia cuando se sostienen afirmaciones y especialmente, cuando se toman decisiones que involucren a los demás.

Así mismo, su labor en aulas implicaría:

- Acompañar a los alumnos en el proceso dialógico: interrogar y cuestionar.

- Proponer normas para el diálogo democrático y hacerles ejercitar todo el tiempo.

- Establecer junto con el grupo los caminos para identificar valores (comenzando por el descubrir).

- Aportar en las formas de indagar nuevos conocimientos (métodos y fuentes de búsqueda).

- Brindar conocimientos teóricos, si es necesario (a través de clases o de intervenciones).

- Promover el debate sobre la priorización de problemas.

- Invitar a identificar posibles vías de solución.
- Interrogar sobre la justificación de las alternativas (razonamiento).

- Promover la argumentación para la defensa pública.

- Ofrecer distintos recursos o caminos para el desarrollo de las competencias requeridas, por ejemplo, a través del arte, en sus diferentes manifestaciones.

\section{RETOS Y PERSPECTIVAS QUE SE PRESENTAN PARA LA FORMACIÓN EN BIOÉTICA}

Entre los más importantes retos que se presentan para el desarrollo de la línea educativa en bioética, se encuentran la escasa investigación en esta disciplina aplicada al área empresarial y la formación en bioética para la docencia ya que su incorporación en la currícula se constituye en un aspecto de transformación educativa.

Finalmente, el clima de una institución a veces se presenta crítico para el cultivo de la reflexión y la deliberación en torno a problemáticas bioéticas. Por ello, se requiere promocionarla desde una política curricular institucional para lograr su aplicabilidad por los docentes y estudiantes.

\section{CONCLUSIONES}

1. La bioética es un marco útil que da soporte a la responsabilidad social porque tiene correspondencia con muchos de sus elementos al incorporar los múltiples actores, el medio ambiente y el ser humano.

2. Las universidades no cuentan con un marco ideológico conceptual que de fortaleza a los planteamientos sobre bioética, como consecuencia sus planes de estudio no la contemplan en su malla curricular, ni los docentes en su gran mayoría, han recibido formación en bioética.

3. Institucionalmente hay una falta de orientación pedagógica clara y definida en este campo.

4. Hay la necesidad de generar las condiciones para lograr un adecuado ambiente de aprendizaje bioético, debiendo los contenidos y estrategias docentes articularse para lograr este propósito. 


\section{REFERENCIAS BIBLIOGRÁFICAS}

1. BEAUCHAMP, T.L.; CHILDRESS, J.F. (1999). Principios de Ética Biomédica. Masson, Barcelona.

2. BUENO, Gustavo (2001). ¿Qué es la bioética? Pentalfa Ediciones. Oviedo.

3. DELORS, Jacques (1989). Una ética del Medio Ambiente. Conferencia pronunciada en Bruselas (Mayo 1989). Reproducida en El nuevo concierto. Europeo. 1993. Madrid. Acento Editorial. Disponible en eciencia.urjc.es/dspace/bitstream
4. FRANÇOIS VALLAEYS, Cristina de la Cruz, Pedro Sasia. (2009). Responsabilidad Social Universitaria, Manual de primeros pasos. McGraw-Hill Interamericana Editores, Banco.

5. POTTER, Van Rensselaer (1971). Bioethics: Bridge to the Future. New Jersey: Prentice Hall.

6. POTTER, Van Rensselaer (1970). Bioethics: The science of survival. 14. 127-153.

7. POTTER, Van Rensselaer (1988), Global Bioethics. Building on the Leopold Legacy, Michigan State University Press, East Lansin.

8. Unión Europea. (2010). Libro Verde. Norma ISO 26000: Guía sobre la Responsabilidad Social. 